\title{
The Role of the Placebo in Clinical Practice
}

\author{
Pesach Lichtenberg*
}

The placebo is the most commonly-employed treatment across cultures and throughout history (1). Today's physician, resting on her evidence-based laurels, might have no trouble accepting this claim when considering the medical practice of yore. After all, what else can one make of the potions, herbs, leechings and rituals of our distant colleagues of an earlier agemedicine men, shamans, wizards - if not that they were, wittingly or ignorantly, purveyors of placebos? None of this ought to be relevant to our enlightened time, when we can exploit our understanding of physiology, pathology, pharmacology and the double-blind randomized placebo-controlled study to deliver scientifically-informed, empirically-validated, precisely-targeted therapeutic interventions.

Yet the placebo is here, a central item of our pharmacopeia, widespread and, certainly in the opinion of most clinicians, effective. This is the clear conclusion of a series of recent studies whose purpose was to gauge the extent of placebo use amongst physicians and to give some sense of how effective practitioners found them to be. Using decidedly low-tech methods (i.e. questionnaires), findings across disparate locales were impressively uniform. For over 500 Danish physicians who responded to a questionnaire, placebo use was as high as $86 \%$ amongst general practitioners, 54\% amongst hospital-based physicians, and $41 \%$ of private specialists (2). In Chicago, $45 \%$ of 231 internists affiliated with three local medical schools admitted to using the placebo (3). In our own study in Israel, we questioned 90 physicians and nurses in primary and tertiary care and found that $60 \%$ used the placebo (4). In all locales, the placebo was administered in a variety of forms and for a variety of purposes, and was believed by most of its purveyors to be ethical and effective.

Some may be surprised by these findings. The local

*To whom correspondence should be addressed:

Pesach Lichtenberg, M.D.

Department of Psychiatry, Herzog Hospital, P.O. Box 3900, Jerusalem 91035, Israel.

E-mail: licht@cc.huji.ac.il. press even typically reports them ("Israeli Physicians Give Phony Meds" was one headline that I found particularly unsavory). Yet it is likely that these reports actually underestimate the extent of placebo use.

Allow me to justify this statement. The placebo is notoriously difficult to define (5). Indeed, even the surveys quoted above did not use a uniform definition. The Danish survey offered as a definition of placebo an intervention "not considered to have any 'specific' effect on the condition treated, but with a possible 'unspecific' effect." The Chicago questionnaire asked the physician to define placebo, the three possibilities including treatment with a non-specific effect (37\%), an intervention not expected to have an effect through a known physiologic mechanism $(51 \%)$, or treatment that is inert or innocuous $(28 \%)$. The Israeli survey simply skirted the issue and started asking questions about placebo use. For all surveys, a placebo could take the form of vitamins, saline injections, sugar pills, antibiotics (for non-bacterial infections), and subtherapeutic dosages of standard medication.

At the risk of reducing one ambiguity to another, it might help to think of the placebo as anything causing a therapeutic reaction, or "placebo effect", by psychological means, such as providing reassurance, assuaging anxiety, eliciting conditioned responses or arousing positive expectancy. (Some may object that I have here consigned psychotherapy to the status of a placebo; but I do not think that is a problem for one who appreciates the potential of placebo effects and the many ways of usefully producing them [6]. Ultimately, psychological effects are as real as any other.)

Thought of in this way, it becomes apparent that the placebo effect is part of every intervention. The inscrutable scrawl on the prescription pad, the reassuring smile, the limp stethoscope hung from the nape of the neck - these all contribute to the placebo effect. So, apparently, do the color of the pill (7), and even the number of pills swallowed by the patient $(8,9)$. You just can't avoid the placebo effect (although, admittedly, comatose patients may be an exception). If you doubt this, just consider what happens if you 
artificially nullify the placebo effect. This has been tried, by concealing from the patient that he was receiving medication. The result was that morphine calmed pain less, diazepam did not calm anxiety at all, atropine only slightly increased heart rate, while propranolol only slightly decreased it $(10,11)$. On the other hand, revealing that the pill to be provided is a placebo may not necessarily abolish its benefit $(12,13)$; apparently our reaction to treatment and all that it entails is too deeply conditioned to be reversed by mere full disclosure.

The placebo is at last being appreciated for its subversive potential. To ponder the placebo leads one to reconsider many of our presumptions about the gap between mind and body, about the untapped subtleties of medical treatment, and about what really heals when we administer our therapies.

People are often concerned about the ethics of the placebo. Certainly, giving a placebo where a more effective therapeutic alternative is available would be unethical. Similarly, providing a placebo in the futile hope of distinguishing "organic" from "supratentorial" maladies, or simply for the purpose of being done with an irksome patient, cannot be defended. Yet under certain circumstances, as I have described elsewhere (14), the placebo - whether an inert pill, a superfluous vitamin, or a miniscule, sub-therapeutic dose of medication - can be legitimately and ethically offered as treatment. If standard treatments have failed or caused intolerable side effects, a placebo may sometimes provide comfort.

Two case vignettes may help illustrate how the placebo can be usefully and ethically employed. A 62year-old man suffering from post-operative pain was treated with repeated intramuscular injections of an opioid analgesic, but continued to complain of intense pain and to demand further injections. The staff, seeking to avoid an excessive dose, decided to alternate opioid analgesics with intramuscular saline. They explained to the patient that saline injections have often been found to alleviate pain, and they expressed optimism that it would help him as well. The patient responded well to the treatment, to everyone's satisfaction.

A 38-year- old woman in psychotherapy for depression expressed her belief that her problem was "chemical", and the talk therapy was pointless. Insisting that she get "a prescription for a pill", the hesitant psychiatrist relented and provided $10 \mathrm{mg}$ of imipramine, a medication that usually requires $100-200 \mathrm{mg}$ and twoto-four weeks to achieve a therapeutic effect. The doctor explained that while higher doses are often used, they would wait to see her reaction to this dose, which can also be of benefit (15). The woman at her next weekly visit reported an immediate and significant improvement. She subsequently decided to leave psychotherapy, returning every 3-4 months to the psychiatrist to renew her prescription.

The ethical problem with the placebo, then, is deceit. The solution, we suggest, is honesty. The physician must convey to the patient the message that, "Though we don't know exactly how this pill works, it can help you feel better."

In fact, if we want to be completely frank with our patients, this sentence might have to accompany just about any treatment we might offer.

\section{REFERENCES}

1. Shapiro AK, Shapiro E. The powerful placebo: from ancient priest to modern physician. Baltimore and London: Johns Hopkins University Press, 1997.

2. Hróbjartsson A, Norup M. The use of placebo interventions in medical practice-a national questionnaire survey of Danish clinicians. Eval Health Prof. 2003;26(2):153-65.

3. Sherman R, Hickner J. Academic physicians use placebos in clinical practice and believe in the mind-body connection. J Gen Intern Med. 2008;23(1):7-10.

4. Nitzan U, Lichtenberg P. Questionnaire survey on use of placebo. BMJ. 2004 23;329(7472):944-6.

5. Gøtzsche PC. Is there logic in the placebo? Lancet 1994(344):925-6

6. Frank, J. D. \& Frank, J. B. Persuasion and healing: A comparative study of psychotherapy (3rd ed.). Baltimore, MD: Johns Hopkins University. 1996

7. de Craen AJ, Roos PJ, Leonard de Vries A, Kleijnen J. Effect of colour of drugs: systematic review of perceived effect of drugs and of their effectiveness. BMJ 1996; 313(7072):1624-6

8. Horwitz RI, Horwitz SM. Adherence to treatment and health outcomes. Arch Intern Med 1993;153(16):1863-8

9. de Craen AJ, Moerman DE, Heisterkamp SH, Tytgat GN, Tijssen JG, Kleijnen J. Placebo effect in the treatment of duodenal ulcer. Br J Clin Pharmacol 1999;48(6):853-60

10. Benedetti F, Maggi G, Lopiano L, Lanotte M, Rainero I, Vighetti S, Pollo A. Open versus hidden medical treatments: The patient's knowledge about a therapy affects the therapy outcome. Prevention \& Treatment. 2003; 6(1)

11. Colloca L, Lopiano L, Lanotte M, Benedetti F. Overt versus covert treatment for pain, anxiety, and Parkinson's disease. Lancet Neurol. 2004; 3(11):679-84.

12. Park LC, Covi L. Nonblind placebo trial: an exploration of neurotic patients' responses to placebo when its inert content is disclosed. Arch Gen Psychiatry. 1965;12:36-45.

13. Vogel AV, Goodwin JS, Goodwin JM. The therapeutics of placebo. Am Fam Physician 1980;22(1):105-9.

14. Lichtenberg P, Heresco-Levy U, Nitzan U. The ethics of the placebo in clinical practice. J Med Ethics. 2004 Dec;30(6):5514.

15. Brown WA. Placebo as a treatment for depression. Neuropsychopharmacology 1994; 10: 265-9

Pesach Lichtenberg heads the Men's Division of Psychiatry at Herzog Hospital in Jerusalem, Israel. He is a Senior Clinical Lecturer at Hadassah Medical School of the Hebrew University of Jerusalem, where amongst other topics he lectures about the placebo. 